\title{
Enhancement of signal detection properties by coupling of active hair bundles
}

\author{
Kai Dierkes*, Benjamin Lindner and Frank Jülicher
}

\author{
Address: Max Planck Institute For The Physics Of Complex Systems, Nöthnitzer Str. 38, 01187 Dresden, Germany \\ Email: Kai Dierkes* - kai@mpipks-dresden.mpg.de \\ * Corresponding author
}

from Eighteenth Annual Computational Neuroscience Meeting: CNS*2009

Berlin, Germany. 18-23 July 2009

Published: 13 July 2009

BMC Neuroscience 2009, I0(SuppI I):P3I5 doi:I0.II86/I47I-2202-I0-SI-P3I5

This abstract is available from: http://www.biomedcentral.com/I47I-2202/I0/SI/P3 I5

(c) 2009 Dierkes et al; licensee BioMed Central Ltd.

In the inner ear of vertebrates, hair bundles transduce mechanical stimuli elicited by sound waves or substrate vibrations into electrical signals. Hair bundles from the sacculus of the bullfrog have been shown to amplify weak periodic stimuli in a frequency selective manner [1]. For stronger stimuli, their responses are marked by a region of non-linear compression [2]. They can also exhibit spontaneous oscillations [3]. These features have been recognized as signatures of the cochlear amplifier. But while the gain of the cochlear amplifier is about 1000 [4], the gain of a single hair bundle is about 10. Single hair bundle dynamics thus cannot account quantitatively for the performance of the cochlear amplifier.

In many inner ear organs, hair bundles are attached to overlaying structures such as tectorial or otolithic membranes, making hair bundles elastically coupled to each other. Here we describe results concerning the effects such a coupling could have on hair bundle dynamics. In a first approach we describe coupling by linear springs. We describe single hair bundle dynamics by means of a biophysical model that has been shown earlier to capture quantitatively the main features of stochastic hair bundle motility [5].

Our simulations show that coupling could, indeed, alter the spectral characteristics of spontaneous movements, rendering stochastic oscillations more coherent [6]. Also, coupled hair bundles can synchronize [6]. As far as signal detection properties are concerned, we find that sensitivity to weak periodic driving and frequency tuning are considerably enhanced when the strength of coupling is chosen in an appropriate way [6].

\section{References}

I. Martin P, Hudspeth AJ: Active hair-bundle movements can amplify a hair cell's response to oscillatory mechanical stimuli. Proc Natl Acad Sci 1999, 96: | 4306- I43 II.

2. Martin P, Hudspeth AJ: Compressive nonlinearity in the hair bundle's active response to mechanical stimulation. Proc Natl Acad Sci 200I, 98: 14386-14391.

3. Martin P, Bozovic D, Choe Y, Hudspeth AJ: Spontaneous oscillation by hair bundles of the bullfrog's sacculus. J Neuroscience 2003, 23:4533-4548.

4. Robles L, Ruggero MA: Mechanics of the mammalian cochlea. Physical Reviews 200I, 8I:I305-1352.

5. Nadrowski B, Martin P, Jülicher F: Active hair-bundle motility harnesses noise to operate near an optimum of mechanosensitivity. Proc Natl Acad Sci 2004, I I I: I 2 1 95- I 2200.

6. Dierkes K, Lindner B, Jülicher F: Enhancement of sensitivity gain and frequency tuning by coupling of active hair bundles. Proc Natl Acad Sci 2008, 105: 18669-18674. 